\title{
Model for the Creation of Mobile Node Knowledge Networks
}

\author{
Chadwick Carreto A., Elena F. Ruiz, Marina Vicario \\ Instituto Politécnico Nacional, School of Computing, Mexico City, \\ Mexico \\ \{carretoa, eruizf, mvicario\}@ipn.mx
}

\begin{abstract}
Knowledge, of any kind or source, is an intangible active, and in consequence, invisible and hard to value. One of mankind's characteristics is his ability to use his own experiences and turn them into actions, which are susceptible of being generalized so they can be transmitted to the following generations. Such natural quality of the individual has transformed into a gradual and systematic process of knowledge, whose purpose has been, amongst others, a higher accessibility to personal development so that it increase the human race's adaptation capacity to the demands of its environment and its social context. In this paper we show the development of a model for creating Knowledge Networks (KNM) networks based on mobile nodes, this model will share relevant and useful for different types of users anywhere, anytime (anytime, anywhere). The KNM aims to make available to users, developing tools for both synchronous and asynchronous communication and to develop the training process with the use of mobile devices with limited capacity Internet connection.
\end{abstract}

Keywords: Knowledge, mobile nodes, knowledge network.

\section{$1 \quad$ Introduction}

Today's society is facing and endless amount of challenges, which include defining better and more timely ways for it to communicate and collaborate. Knowledge is the most important variable in any organization, without leaving aside the importance of material, technological or financial resources, it is currently considered as one of the most benefiting or prejudicial factors.

This situation requires models and ways to communicate and above all, share and increment knowledge to face the changes demanded by organizations. Changes with a focus on integral development, considering that traditional and current administration models are in a critical phase, in which they cannot resolve problems or satisfy the needs of today's society; it's necessary to migrate to Knowledge Management solutions and create networks that allow the transfer and exchange of knowledge.

However, most specialized authors agree in defining knowledge management based on the different stages that make it up and/or in its objectives [2]. 
Knowledge Management has the capacity to generate knowledge and induce learning [4] define it as the ability that people have to understand and manage information using technology and knowledge sharing.

In the globalized society of the twenty first century, knowledge networks constitute the maximum expressions of man as a knowledge producer and his need to exchange and transfer what he learns and creates, based on the social interaction in a technological platform given a very particular context.

Knowledge production is closely related to the information needs and the formal organizations created for that purpose. This integration producing knowledge based on society's integral needs as its objective. The main challenges are: Communication, Collaboration and Sharing (CCS).

Given this issue, it is important for organizations to implement knowledge management models and networks to allow their staff development through schemas that allow sharing and generating knowledge in an easy, practical and cheap way.

The structure of the paper is defined in the following way: Section 2 describes the context and the Model proposal to create Knowledge Networks; section 3 describes the technology that was used for this, section 4 identifies the implementation of the model with its results and finally section 5 presents the conclusions and future work.

\section{Knowledge Network and the Context}

We shall define a Knowledge Network as a community of people, who in a formal or informal way, either part or full time, work for a common interest and base their actions in the formation, development and sharing of new knowledge [1].

In a more social and dynamic context, knowledge networks are the human interactions that produce, store, distribute, share, access and analyze knowledge produced by mankind in a systematic way or by personal or group interest of sharing any kind of data by any means, generally electronic; in order to develop their capacities for creating, understanding, power, study and transformation of reality surrounding territory in a given social and economic context [5]

As mentioned earlier, knowledge networks are the result of the human activity essentially formed by producing, managing and transferring knowledge and largely structured by organizations created for that purpose. In this regard, there are several models aimed at the technological and social.

In this fashion, some authors, such as Andreoli [2] propose a knowledge network model with a technological approach based on three fundamental elements: a central core, collaboration clusters and knowledge generation units. They state that a network is made up of an entity or groups of people who lead or coordinate activities aimed towards knowledge generation making use of previously defined topics, then generate groups or research facilities where and analogous network can group a network or more of them. Electronic communication media mainly gives support of this integration.

Also, Atwell propose a model of knowledge networks based of the same technological standpoint but directed to knowledge management in the so-called virtual communities [3]. This model is structured by three basic elements: the network 
members, the network tools made up by a pure information network and the discussion topics defined by the members (people or organizations).

These experiences, one being Latin American and another of the European Union, enrich the subject of knowledge networks through different schemas that share two fundamental variables: a group of people who live in a society and a technological platform which optimizes knowledge production and transfer made by the former.

In this proposal, knowledge networks are based in a knowledge node interconnection model, which can be translated into a mobile collaboration network that is more focused in its social and organizational character. In this sense, knowledge networks are made up by different kinds of networks, e.g. social networks, main networks, institutional networks, collaboration networks and transfer networks.

Social networks are inserted in the conception of the collaboration action theory, which defines that "social capital made by reciprocity, voluntary cooperation and commitment" [1] are part of the social system dynamics

Social networks share different types of information, data, knowledge and assets. In the same way, they involve mutual benefit, trust and coordinated work that are intimately mediatized by the globalized information world.

The basic primary networks are divided into social action network, which are "the sum of management, administration, participation or association relationships that span the plurality of people or micro-organizations" [1]

Institutional networks are all the organizations created or organized to produce knowledge by researching the needs of social networks and/or the problems of the primary networks, to help their development and social advancement. University, research institutes, and technological development facilities either private or public, among others, compose these networks. Social action networks also operate in this kind of networks.

This networks operate by the thousands in every country around the globe, so that there can be various institutions in the same country researching the same problem without being dynamically and effectively integrated in order to save time, money and effort. Because of this problem, collaboration networks were formed.

Collaboration networks are public or private organization in a local, regional and international level, which can cooperate technically and financially with the institutional networks to manage joint product under relevancy, efficiency, productivity and development parameters.

On the other hand, transfer networks are known for grouping entities, people or organizations that have as a purpose mediating or translating knowledge into social networks and are intimately bonded to the technological innovation and knowledge development processes.

The purpose of this paper is to contextualize how this kind of networks could work together making use of current technology and based primarily on information exchange media that are becoming more common, such as mobile devices.

Technology and telecommunications allow creating node networks that are no more that simple users exchanging information and which can be extended to networks of people sharing knowledge. 


\section{Model of Knowledge Network Creation}

In the real world, individuals share information. According to their characteristics and their need to work with this information, they can be classified in groups of interest and knowledge. In this case, the defining characteristics are the individual needs and the way they interact with other Knowledge Network elements. Under this schema, the networks are characterized by the kind of users that interact among themselves. For example, an academic knowledge network will be composed by students, teachers and researchers with particular interest, each of them with restrictions and a way to interact with other entities of this network.

Along this line, as shown in Figure 1, networks are defined by the sets of entities that belong to different knowledge groups and the sets of media which they use to interconnect and make use of sharing, classification and knowledge sharing services or any kind of service. The set made up by all the elements that exist in a $\mathrm{KN}$ will be denoted as $\mathrm{S}$ and the set of all the types of interconnection media as A.

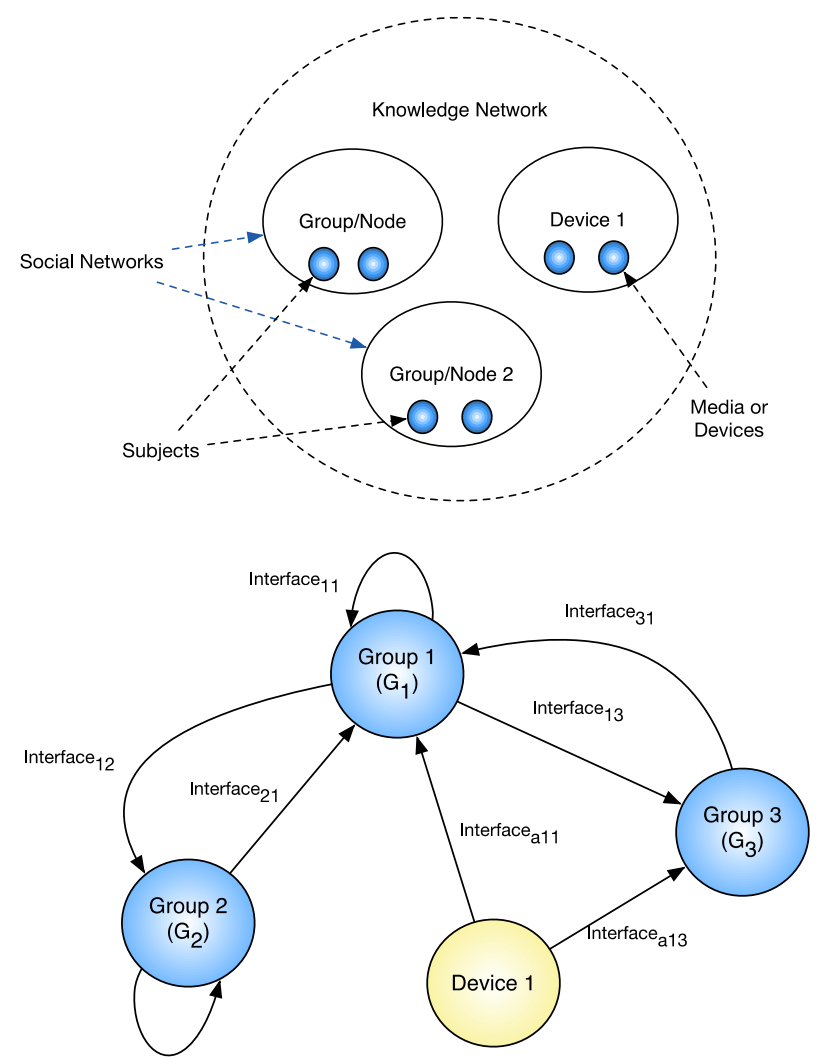

Fig. 1. Node Network Components where a user group collaboration schema.

When a group inside the $\mathrm{KN}$ is defined, their information sharing capacity along with the media the entities belonging to this group will possess, and the set of services that they can provide to other entities when forming an interaction must also be defined. 
Then, each of this groups define in a partial form, the way in which it's going to interact with the rest of the groups, because it defines the interchange actions and the knowledge generation that it can do in favor of the entity with whom it interacts.

To define the interactions that can be done in an KN, we can use a directed graph:

$$
G=(V, E) \text { where } V=S \quad A \text { y } E \subseteq S \cup A \times S .
$$

The edges represent a service relationship, that is, as shown on Figure 1, if an edge gets out of a group G1 and goes into a group G2, indicates that and entity belonging to G1 will offer a service interface to an entity belonging to G2 at the moment a collaboration is done. Similarly and due to an edge existing from G2 to G1, an entity belonging to $\mathrm{G} 2$ will offer a service interface to an entity belonging to $\mathrm{G} 2$ at the moment of collaboration. We shall define the process of executing an action defined in the collaboration interface exposed by another individual as knowledge generation action.

Every edge of $\mathrm{G}$ defines a particular collaboration interface; this allows an individual to collaborate in a particular way depending with whom it interacts. Using the academic network example, a student will present different collaboration interfaces when interacting with another student or a teacher. These interfaces must be defined when designing a $\mathrm{KN}$ and as shown later, will be reflected in the methods of knowledge generation as in components that will be used to call the services of the same networks.

The definition of $\mathrm{E}$, the devices have the particularity that the information exchange is direct among entities or other devices and allows them to update with valuable and timely information that can be characterized as knowledge. This is due to the fact that artifacts are conceptualized as reactive entities that execute activities in response to request from the entities, but that are also available and updatable anytime, anywhere. As the result of using the services exposed by a device, an entity can make use of knowledge and information in an automatic way, making it aware of the status of some other entities or group or complete network, relational databases with the groups, with another entity or with any aspect modeled in the KN.

As we said earlier, the relationships defined in the graph are translated into graphic interfaces that will be shown on the user's mobile devices that belong to a KN when the user triggers an information request event. A wide spectrum of collaboration events can be defined, from which the simplest of them gets triggered when two entities of the same group share all their information and filter it according to their needs.

In a graphical way, inside the interaction graph, the names of the entities that the entities will deploy to collaborate with their peers and the interfaces that must be implemented by the entities if they required modifying their status as a result of a knowledge generation action can be defined. For example, if a user I1 belonging to G1 gets integrated into a knowledge group or to another user I2 belonging to G2, this

will make that both individuals exchange their collaboration interface, so that 2 will send I 2 an Interface 21 and I1 will send I2 an Interface12. When the individuals do the requested interaction, it is assumed that collaboration has ended and the graphical interfaces representing Interface 21 and Interface 12 disappear from devices I1 and I2 respectively. 


\section{Implementation and Results}

The model is designed for mobile systems and basically in node networks (Figure 2 shows the architecture), where it is defined that each user has access to one or more interconnection devices and that the groups that generate one or more knowledge networks are connected using interconnection domains. This is done using various Wireless Technologies that can operate between them, so that this is not an obstacle when communicating. For practical purposes we worked with devices connected to a IEEE $802.11 \mathrm{~g}$ standard Wi-Fi network. The end-user interaction is fundamental for the model, because that's what makes the user comfortable and completely familiarized with the service management in addition to offering them in the time and manner in which they are requested.

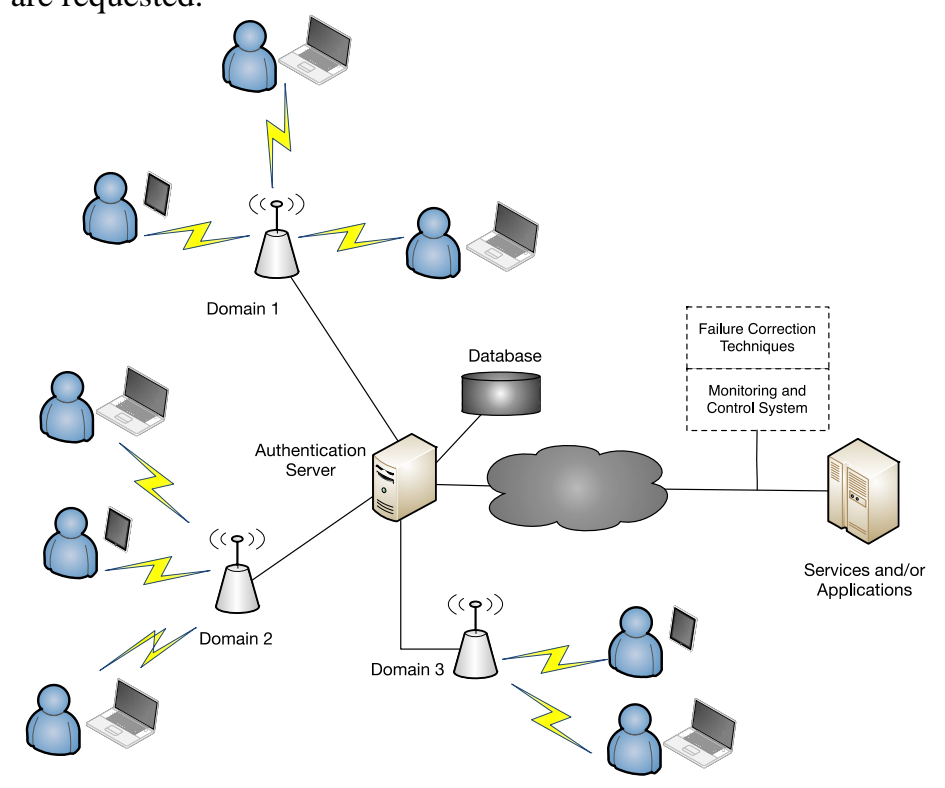

Fig. 2. Architecture of the Model Implementation.

We will briefly describe the process that a service request must follow so that the existing services in a Network can be assigned to the end-users.

- The user, through its device, and an interconnection device from the Network domain exchange information to establish an information request in the knowledge network

- Then the user and its interest groups are validated and verified with the purpose of integrating information of its database to the social database and filtering the user's preferences.

- The information that was found according to the defined criteria is once again filtered in a semantic context to integrate just the relevant information according to the needs of the requesting user, when the user needs it.

- Right away, a server will be in charge of offering a list of services and application associated to a user profile database. 
- The user must select one of the available services and applications. For this, a connection with the servers where the filtered and defined information is established, so that it can be processed and delivered to the user.

- Software will monitor and control job status and the interaction between nodes based on the aforesaid work model.

The model implementation case study was developed in the ESCOM-IPN, which is an academic unit belonging to the IPN that trains Computer System professionals in undergraduate and postgraduate levels. It has a student body of 4000 undergraduate students and 50 postgraduate students. The educational offer in the postgraduate level is a Master of Science Degree on Mobile Computational Systems.

The case study implementation and tests of the model were done in the "Application Development for Mobile Devices" subject, with the help of 40 undergraduate students and in the postgraduate lab.

Testing was done to measure the ratio of participation and information, resource and service use of the "Application Development for Mobile Devices" students, specifically on the "Android Application Development" topic. We looked for a greater degree of development in programming competences using systems and the knowledge network implementation.

The group was examined on the concepts of Java development for Android version 2.0, the educational model was based on live lessons and practices guided and evaluated during a 4 week span, which generated a set of practices and a test where the academic performance of the 40 students was evaluated.

Using said results; students were given credentials to access the Academic Knowledge Network System (figure 3) so they could have information on the subject (Application Development for Mobile Devices) according to their requirements at the moment they needed it, using any mobile device with Internet access.

In the next test based over the same topic (Java development for Android OS), but now on version 2.2, which implies a change of libraries and programming logic for sensors and accelerometers; the educational model was based on counseling given by the Facilitator, the system was in charge of giving the students information on the libraries and technical bases for software development when they needed them and it was also in charge of following up with the proposed practices in a span of 4 weeks.

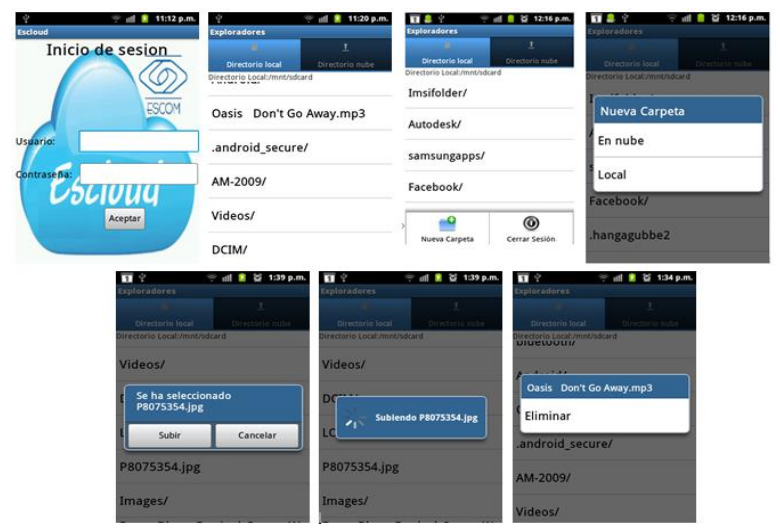

Fig. 3. Interface of the System used for the Model Implementation 
The obtained results helped us observe how the students generated a series of statements between them based on the information contained in the core documentation and the updated information generated via the collaboration and interaction of the users within the system. The system used for this implementation could be tested on different platforms, due to it being developed for iOS, Android and having a responsive interface developed in HTML5, compatible with the majority of browsers and devices.

The model was also tested with different knowledge networks, made up by a group of entities and their devices. In these tests, service domains of a network were split and a network was created for each communication domain. Communication between entities was done by sharing their knowledge bases, but most importantly; by sharing experiences and information sharing and by the interaction between groups and entities.

The main test defined to work with different networks was designed to interconnect different areas of the Instituto Politécnico Nacional so that they could share information and services in a Knowledge Network composed by 3 Academic Units. Each Academic Unit had a set of users and each user had one or more interconnection devices. Each user registered his/her device on the system and could access to it, in a way that a group was form given the user's mobile location and domain. A node that shared information and services was formed by each of the users, which in turn generated a knowledge base.

Once the groups were defined, communication was established between them and the knowledge base generated by the community was shared.

According to the gathered results, we propose that the project can be applied to a greater amount of nodes. According to technological evaluations and studies, an average of 600 to 1000 simultaneous online nodes or 1000 to 5000 offline nodes can be supported by a simple and basic technological infrastructure. This due to the low hardware requirements, simple communication in which entities don't have to spend a lot of resources and the fact that every day the resources get cheaper.

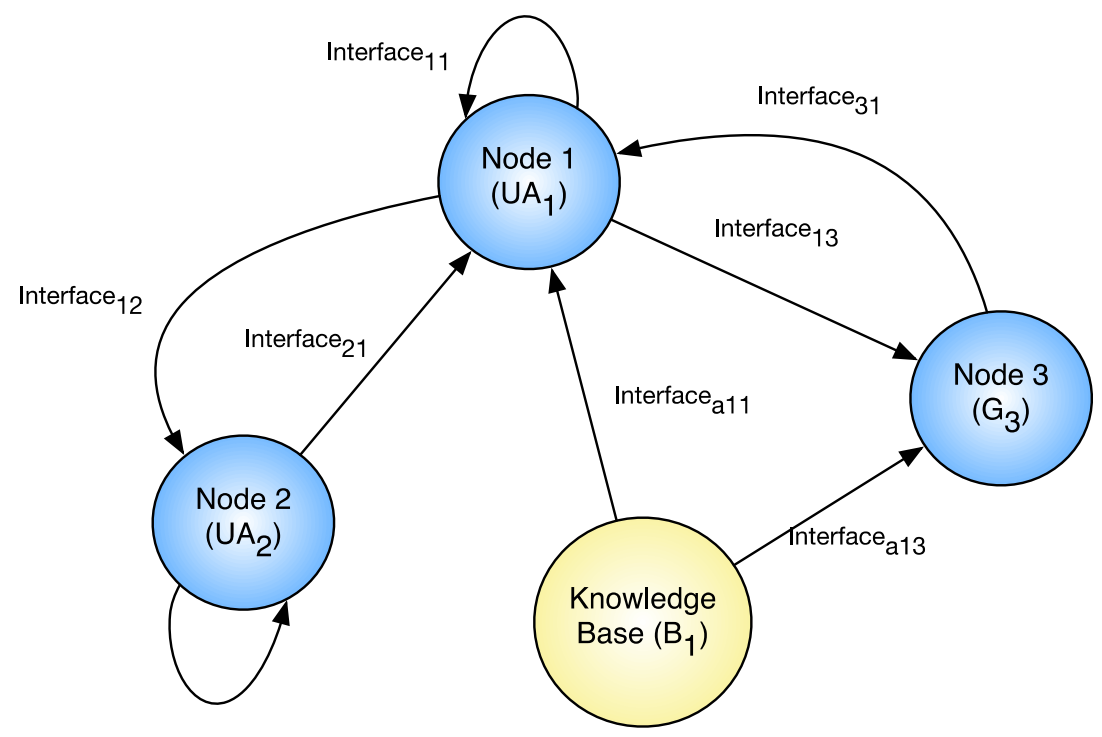

Fig. 4. Interconnection between Test Nodes. 


\section{$5 \quad$ Conclusions and Future Work}

The main contribution of the proposed model is the mobility, which translates to saving time and effort in the knowledge network generations. The model itself is another way to help people do their work in any area they desire, not only because it eases work, but because it gives access to well defined, relevant and explicit information when it is required.

The gathering, synthesizing, reflecting and discussing of information is essential for knowledge management; technology has to support all four activities. Knowledge will never be definite. It's incubated and grows without end.

It is important to test the amount knowledge acquired by the use of the proposed model with different users.

The proposed model is a product of a research project that involves two important aspects of information technologies: Knowledge networks and service nodes communication networks.

In such an environment, users can be in constant interaction, which brings feedback, encourages discussions and makes reaching goals a more agile process. Computing technologies, on the other hand, provide us the capacity of bringing the environment to any physical place and in any moment; removing the need to be in front of a fixed computer to be able to access the system.

The kind of architecture that was developed can be useful for other purposes. Thereby, an important conclusion that we have reached is that the architecture if fully flexible and applicable to multiple areas and different user needs. Then, applicability of the architecture can be changed in a simple way.

One way that the architecture could be substantially improved would be creating a cloud that allows storing and sharing the knowledge base with the linked services to all users.

As future work, it is important to highlight that this proposal can be implemented over diverse research fields and education; but the possibility of it being implemented on information distribution areas like economics, politics, social and cultural areas is not dismissed.

Acknowledgements. The authors thank the support given to this paper, especially by IPN, ESCOM, CIC, UPIICSA, COFAA, SIP and ICyT DF for the facilities given to conduct our research.

\section{References}

1. Adell, J.: Sobre Entornos Personales de Aprendizaje de Universitat Jaume I. http://files.competenciasbasicas.webnode.es/200000168-105691150b (2009)

2. Hewell, H.: eLearning now. eLearning Papers, 2 (1), Barcelona: elearningeuropa.info. Retrieved (December 2014)

3. Attwell, G.: Personal Learning Environments - the future of eLearning? eLearning Papers, 2 (1), Barcelona: elearningeuropa.info. Retrieved December 18, http://www.elearningeuropa.info/out/?doc_id=9758\&rsr_id=11561 (2012) 
Chadwick Carreto A., Elena F. Ruiz, Maria Vicario

4. Dutta, S., De Meyer, A.: Building Assets in Real Time and in Virtual Space. Knowledge Management INSEAD, Denmark (2007)

5. Kalz, M.: Building Eclectic Personal Learning Landscapes with Open Source Tools. In: Conference proceedings for the Open Source for Education in Europe, Research \& Practise conference, Open University of the Netherlands, Heerlen, Retrieved December 18, 2008, http://www.openconference.net/viewpaper.php?id=16\&cf=3 (2008) 